\title{
Quellen zur Geschichte der Neuen Frauenbewegung im Archiv des Instituts für Zeitgeschichte
}

\author{
Eine Zwischenbilanz nach zehn Jahren
}

Archive sind traditionell die wichtigste Fundgrube für die historisch arbeitenden Wissenschaften. In den dort verwahrten amtlichen Akten, in Nachlässen und Verbandsschriftgut finden Historikerinnen und Historiker zeitgenössische Unterlagen zu ihren Fragestellungen. Dies gilt auch für die historische Frauen- und Geschlechterforschung. Denn in fast allen Archiven lassen sich Quellen finden, in denen sich Erfahrungen und Aktivitäten von Frauen widerspiegeln. Da jedoch auch Archivarinnen und Archivare bei der Auswahl und Beschreibung der ihnen anvertrauten Materialien stets von den gesellschaftlichen und kulturellen Wahrnehmungen ihrer eigenen Gegenwart geprägt sind, wurden Unterlagen von und über Frauen in den traditionellen Archiven lange Zeit nicht systematisch gesammelt. Die in allen Beständen vorhandenen Dokumente zu Frauen, ihren Anliegen und Schicksalen verbergen sich zudem meist hinter allgemeinen Erschließungsinformationen und sind so für die Wissenschaftlerinnen und Wissenschaftler nur mit großem Aufwand aufzufinden.

Um die Defizite in der Überlieferungsbildung der öffentlichen Archive auszugleichen, gründeten Aktivistinnen seit den 1970er Jahren zahlreiche Spezialarchive, die meist unabhängig von den öffentlichen Archiven und häufig ehrenamtlich geführt werden. Die Spezialarchive sammeln Unterlagen von und über Frauen, bereiten sie auf und machen sie zugänglich ${ }^{1}$. Nach und nach wuchs auch bei den staatlichen und kommunalen Archiven ein Bewusstsein für die Wichtigkeit einer gezielten Sammlungstätigkeit in Ergänzung zur amtlichen Überlieferung und mittlerweile finden sich auch dort zahlreiche Bestände zur Geschichte von Frauen ${ }^{2}$.

Das Archiv des Instituts für Zeitgeschichte, das sich seit nunmehr über zehn Jahren verstärkt darum bemüht, Unterlagen zur Neuen Frauenbewegung zu akquirieren, veranstaltete am 16. Juli 2015 einen Workshop mit dem Titel „Wie wird aus Aktion Geschichte? Von der Frauenbewegung ins Archiv und in die Geschichtsbücher" mit Vertreterinnen und Vertretern einschlägiger Bewegungsund Spezialarchive, staatlicher und kommunaler Archive sowie Teilnehmerinnen aus Wissenschaft und politischer Frauenbewegung. Einmal mehr bestätigten die Diskussionen, dass in der Regel nur Persönlichkeiten, Initiativen und Organisationen in der Forschung berücksichtigt werden und damit mittel- und langfristig Eingang ins historische Bewusstsein unserer Gesellschaft finden, deren Unterlagen in Archiven und ähnlichen Einrichtungen zugänglich sind.

\footnotetext{
${ }^{1}$ Die 40 im Dachverband „ida“ zusammengeschlossenen Bibliotheken, Archive und Dokumentationsstellen in Deutschland, Österreich, Luxemburg, Italien und der Schweiz zeigen und bewahren Lesben- und Frauengeschichte; vgl. www.ida-dachverband.de.

${ }^{2}$ Beispielhaft dafür: Stadtarchiv München, Bestand Archiv der Münchner Frauengesundheitsbewegung.
} 
Da das IfZ-Archiv sowohl umfangreiche Aktenbestände national und international agierender Organisationen und Initiativen als auch kleinere Bestände mit lokaler oder regionaler Bedeutung aufbewahrt und erschließt, leistet es einen wichtigen Beitrag bei der Dokumentation der Vielschichtigkeit und Pluralität der „Neuen Deutschen Frauenbewegung“. Mittlerweile ist ein umfangreicher Quellenbestand vorhanden, der der interessierten Forschung zur Verfügung steht. Über Sammlungen und Nachlässe, Druckschriften und graue Literatur haben Wissenschaftlerinnen und Wissenschaftler Zugang zu Unterlagen unterschiedlicher politischer Vereine und Organisationen, karitativ arbeitender Gruppen, Frauengruppen von Großorganisationen (etwa verschiedener Frauengremien bayerischer Gewerkschaften) sowie Vor- und Nachlässen, beispielsweise dem Bestand Cornelia Lohmeier, einer vormaligen Funktionärin der Deutschen Jungdemokraten ${ }^{3}$. Die primär für Frauen- und Geschlechtergeschichte infrage kommenden Bestände des Archivs umfassen bereits mehr als einen laufenden Kilometer Akten und reichen chronologisch von der unmittelbaren Nachkriegszeit bis in die Gegenwart. Obwohl der regionale Schwerpunkt in München und Bayern liegt, decken die enthaltenen Unterlagen im Grunde alle Facetten der Neuen Frauenbewegung in der Bundesrepublik ab ${ }^{4}$. Damit kann bereits ein Gutteil ihrer Forderungen, Erfolge und Misserfolge aus verschiedenen Perspektiven untersucht werden. Es lassen sich sowohl die internen Beziehungen verschiedener Gruppen und Initiativen als auch deren Verhältnis untereinander sowie Verbindungen und Netzwerke in der Münchner Gesellschaft nachvollziehen und mit Aktivitäten an anderen Orten in Bezug setzen ${ }^{5}$.

Zur sozialgeschichtlichen Untersuchung eines typischen Frauenberufs findet sich etwa in dem knapp 600 Akteneinheiten umfassenden Bestand des Berufsver-

\footnotetext{
${ }^{3}$ Vgl. folgende Bestände im Archiv des Instituts für Zeitgeschichte (künftig: IfZ-Archiv): Monika Lochner-Fischer (Signatur ED 751), Frauenakademie München (ED 892), Arbeitsgemeinschaft sozialdemokratischer Frauen (ED 894), Frauenbewegung München (ED 899), Cornelia Lohmeier (ED 457), Hildegard Hamm-Brücher (ED 379), Gewerkschaftsfrauen München (ED 897), Verein für Fraueninteressen (ED 898), Deutscher/Bayerischer Frauenring (ED 893), Inge Aicher-Scholl (ED 474), Christel Küpper /Friedensbewegung (ED 702), Rijk Hilferink Flugblattsammlung (ED 735), Helke Sander (ED 914), Hannelore Mabry/Bayer. Archiv der Frauenbewegung (ED 900). Im Bereich Sammlungen sind weiter zu nennen: Papiere der Arbeitsgemeinschaft der Wählerinnen (Signaturen ED 486 und Dn 100) oder W.O.M.A.N. Weltorganisation der Mütter aller Nationen in versch. Verbandsschriftgut und in Dp 829. Weiter liegen Druckschriften von über 90 Berufs-, Sozial- und politischen Frauenorganisationen vor, unter anderem aus Frauenzentren bundesweit mit den Signaturen Dp 849-858.

${ }^{4}$ Vgl. Ute Elbracht, Neue Soziale Bewegungen - ein Sammelschwerpunkt des Archivs des Instituts für Zeitgeschichte, in: VfZ 59 (2011), S. 149f.; IfZ-Archiv, Bestand Helene Weber/Mitarbeit im Parlamentarischen Rat 1948f. (ED 160) bis zu Bestand Frauenakademie München u. a. Wiedereinstiegsberatung für Frauen in das Berufsleben, Tätigkeiten bis 2007 (ED 892).

${ }^{5}$ Ergänzende Überlieferungen finden sich z. B. im Archiv der deutschen Frauenbewegung Kassel, im Frauenforschungs, -bildungs- und dokumentationszentrum Berlin, im Archiv für alternatives Schrifttum Duisburg, im Frauenstadtarchiv Dresden, im Archiv der Münchner Arbeiterbewegung, im feministischen Archiv Marburg, im FrauenMediaTurm in Köln, im Archiv der sozialen Demokratie in Bonn und in vielfältiger Weise in den staatlichen und kommunalen Archiven sowie in den verschiedenen Universitätsarchiven.
} 
bands katholischer Arbeitnehmerinnen in der Hauswirtschaft (bkh) vielfältiges Material. Die Mitglieder dieser gewerkschaftlichen Bewegung arbeiteten überwiegend in Einzelanstellungsverhältnissen und entwickelten eine ganz eigene Art der beruflichen Interessenvertretung. Sie waren darüber hinaus europa- und sogar weltweit vernetzt. In den internen Protokollen und Aufzeichnungen der Landesverbände ist beispielsweise die transnationale Kooperation des Verbands mit anderen nationalen und internationalen Organisationen dokumentiert, etwa mit TraNoi, dem Verband italienischer Hausangestellter, mit der Internationalen Arbeitsgemeinschaft Katholischer Hausgehilfinnenverbände (IAG) oder auch der Weltorganisation der Katholischen Frauenorganisationen UMOFC ${ }^{6}$. Ein zentraler Arbeitsschwerpunkt des bkh war die Professionalisierung der Hausarbeit, die durch wechselnde gesellschaftliche und politische Rahmenbedingungen starken Veränderungen unterworfen war, welche sich ebenfalls im Bestand nachvollziehen lassen. Der Berufsverband für katholische Hausangestellte nahm auch an Debatten über Rollenbilder von Frauen, Aufgaben und Zuschreibungen von Familienarbeit und damit an der gesellschaftlichen Meinungsbildung zum Thema „Lohn für Hausarbeit“ teil ${ }^{7}$. In diesem Zusammenhang sind zudem die innovative Rolle des bkh bei Tarifverhandlungen für die Mitglieder bzw. das Beschäftigungsklientel, das Engagement bei der Einführung von Berufs- und Weiterbildungsangeboten und die Entwicklung von hauswirtschaftlichen Beratungsangeboten zu nennen. Auch jüngste Entwicklungen spiegeln sich in den Verbandsunterlagen wider. Dazu gehört der Anstieg von Privatinsolvenzen, die zunehmende Zahl von Anträgen auf Leistungen nach dem Sozialgesetzbuch II sowie veränderte Konsum- und Angebotskulturen, die neue Interpretationen und eine erweiterte historische Perspektive auf Hausarbeit fordern. Das betrifft etwa die Reduktion von hauswirtschaftlichem Unterricht an Schulen, den völligen Wegfall von Ausbildungsabschnitten über Haushaltsführung und Ernährungswissenschaft - dem gegenüber stehen Convenience-Lebensmittel und eine Ausbreitung von Lieferservicen nicht zuletzt in Schulen und Kinderbetreuungseinrichtungen, was in die Familien zurückwirkt. Nicht vergessen werden dürfen Diskussionen und aktuelle Forschungsansätze im Bereich der (Familien)Pflege. Hier geht der Bayerische Forschungsverbund „Gender \& Care“ (ForGenderCare) ${ }^{8}$ beispielhaft voran, der

\footnotetext{
${ }^{6}$ IfZ-Achiv, ED 895, Bestand bkh, Laufzeit (1906) 1920-2007. Zur internationalen Zusammenarbeit z. B. mit UMOFC, IAG, TraNoi. Movimento Lavoratrici della Casa, Verband katholischer Hausangestelltenvereine Schweiz, Verband der christlichen Hausgehilfinnen Österreichs, Cultura Orientacion y Prevencion para el Servicio (COPS, Barcelona), Bildungszentrum für Hilfe und Orientierung für Hausangestellte (San Jose, Bogota), Sindicato Livre de Empregadas Domesticas (Lissabon); darin ebenfalls Materialien zur Zusammenarbeit mit der Amtskirche und den Laienorganisationen. Ein Schwerpunkt der Überlieferung bezieht sich auf Berufs- und Bildungsangebote im Bereich Hauswirtschaft seit den 1950er Jahren bis hin zu Verhandlung von Tarifverträgen.

${ }^{7}$ Ifz-Archiv, Mabry/BAF (ED 900, Bde. 65, 127, 289, 290, 337), und Neue Frauenbewegung (ED 899, Bde. 2, 3, 9, 11).

${ }^{8}$ Vgl. www.forgendercare.soziologie.uni-muenchen.de/index.html.
} 
das Thema Hausangestellte ganz neu beleuchtet. Auch dazu kann der Bestand bkh aus historischer Sicht interessante Einblicke liefern.

Andere sozial- und gesundheitshistorische Auswertungsmöglichkeiten bieten Unterlagen über eine besondere Aktivität des Stadtbunds Münchner Frauenverbände, den monatlichen Telefondienst „Aktuelles aus dem Gesundheitswesen“. Unter einer zentralen Rufnummer der Deutschen Bundespost konnte zwischen 1970 und 1990 eine Beratungsansage abgerufen werden, die über Themen rund um Familie, Elternschaft, Umweltbelastung im Haushalt, Ernährung, später auch zum Thema „AIDS/HIV“ aufklärte. Der Stadtbund holte staatliche Stellen mit ins Boot, namentlich bayerische Ministerien und Gesundheitsämter, um fundiert und leicht erreichbar informieren zu können; überliefert sind sowohl die Skripte der Ansagen wie auch umfangreiche Statistiken zur Nutzung 9 .

Mit der Bezeichnung „Schwesterstädte“ umschrieb der Stadtbund sein Engagement im Bereich der internationalen Städtepartnerschaften - auch Jumelage genannt - seit Anfang der 1960er Jahre. Der Archivbestand hält auch dazu zahlreiche Unterlagen bereit. Sie zeigen, wie kommunale Politik von zivilgesellschaftlichen Akteurinnen mitgetragen und der Demokratisierungsgedanke der Jumelage mit Leben erfüllt wurde. Der Stadtbund engagierte sich unter anderem im Kontakt zu den Partnerstädten Bordeaux, Verona und Edinburgh ${ }^{10}$. Er organisierte den Versand von Städtefreundschaftskarten, eigenständige Besuchsreisen und hielt Kontakte zu den dortigen Frauenorganisationen. Regelmäßige Begegnungen des Stadtbunds mit Frauendelegationen aus verschiedenen Städten Japans und der Sowjetunion sind im Bestand ebenfalls ausführlich dokumentiert. Es gab in knapp dreißig Jahren vielfältige Verbindungen, unter anderem gemeinsames Gedenken an die Atombombenabwürfe 1945 und das Ende des Zweiten Weltkriegs ${ }^{11}$. Die Auswertung insbesondere der Unterlagen zu sowjetischen Frauendelegationen verspricht interessante Einblicke in systemübergreifende Kontakte.

Fragestellungen, welche die Politisierung von Frauen verschiedener sozialer Gruppen, die Entwicklung, Artikulation und gesellschaftliche Etablierung von Forderungen, die Professionalisierung von Verbandsarbeit sowie verschiedene Formen politischer Lobbyarbeit berühren, können anhand einer Vielzahl von Beständen im IfZ-Archiv untersucht werden. Da in Vor- und Nachlässen, die das IfZ-Archiv verwahrt, Münchener Gleichstellungspolitik seit den frühen 1970er Jahren überliefert ist, sind auch vergleichend oder beziehungsgeschichtlich angelegte Studien möglich ${ }^{12}$. Schließlich können anhand des politischen Nachlasses der SPD-Landtagsabgeordneten Monica Lochner-Fischer (1952-2012) und der Unterlagen der großen Frauenverbände die Umsetzung von politischen Forderungen in Gesetze und Verordnungen untersucht und darüber hinaus die Hand-

${ }^{9}$ IfZ-Archiv, ED 896 (Stadtbund Münchner Frauenverbände), Bde. 88-106.

${ }^{10}$ Ebenda, Bde. 139-143.

${ }^{11}$ Ebenda, Bde. 144-154.

12 Zur Gleichstellungsstelle der Landeshauptstadt München vgl. etwa die folgende Bestände des IfZ-Archivs: Stadtbund Münchner Frauenverbände, Frauenakademie München, Mabry/ BAF und Verein für Fraueninteressen. 
lungsspielräume staatlicher Verwaltung vermessen werden. Kontakte in ministerielle Vorzimmer und diverse Verwaltungsebenen waren und sind unerlässlich, um erfolgreiche Lobbyarbeit zu leisten - inwieweit die bayerische Verwaltung den Ideen der Frauen positiv oder eher ablehnend gegenüberstand, wer Türen öffnete oder schloss - all dies steht in den Akten ${ }^{13}$.

Ute Elbracht, Esther-Julia Howell

${ }^{13}$ Beispielhaft sei hier auf die Verwaltungsjuristin Christine Marino verwiesen, erste Leiterin der Leitstelle für die Gleichstellung von Frauen und Männern im Bayerischen Staatsministerium für Arbeit und Sozialordnung, Familie und Frauen. 


\section{GRUNDLAGENWERK ZUM ALLDEUTSCHEN VERBAND IN DER WEIMARER REPUBLIK}



Uta Jungcurt

ALLDEUTSCHER EXTREMISMUS IN DER WEIMARER REPUBLIK

Denken und Handeln einer einflussreichen bürgerlichen Minderheit

2016, X, 396 S.

Geb. $€ 69,95$ [D]

ISBN 978-3-11-045477-2

eBook € 69,95 [D]

PDF ISBN 978-3-11-045749-0

ePUB ISBN 978-3-11-045518-2

Print + eBook $€ 109,95$ [D]

ISBN 978-3-11-045750-6

Der Alldeutsche Verband spielt in der Forschung eine zentrale Rolle-allerdings nur für das Kaiserreich und den Ersten Weltkrieg, während er in seiner verhängnisvollen Bedeutung für die Weimarer Republik kaum wahrgenommen worden ist. Ganz zu Unrecht, wie Uta Jungcurt in einer breit recherchierten Studie eindrucksvoll und mit viel Sinn für das sprechende biographische Detail zeigen kann. Der Alldeutsche Verband - eine Vereinigung nationalistischer Bildungsbürger - kämpfte von Beginn an gegen Weimar und erwies sich dabei als Stichwortgeber und ideeller Steigbügelhalter der NSDAP, der er auch ideologisch immer näher rückte. Vor allem die Übernahme und Propagierung extremer völkisch-rassistischer Positionen bahnte Hitler den Weg. Auch dem Alldeutschen Verband waren ideologische Fernziele wie ein völkischer Staat oder eine völkische Diktatur nicht fremd.

Uta Jungcurt, Universität Mannheim. 